\title{
Formal Equivalence Analysis
}

\author{
Francisco J. Valverde-Albacete ${ }^{a}$, Carmen Peláez-Moreno ${ }^{a}$, Pablo Cordero ${ }^{b}$ and \\ Manuel Ojeda-Aciego ${ }^{b}$ \\ ${ }^{a}$ Depto. Teoría de Señal y Comunicaciones, Univ. Carlos III de Madrid, Madrid, Spain, \{fva,carmen\}@tsc.uc3m.es \\ ${ }^{b}$ Dpt. Matemática Aplicada, Univ. de Málaga, Málaga, Spain, \{pcordero,aciego\}@uma.es
}

\begin{abstract}
Following R. Wille's lead and suggestion we set out to design a new kind of view onto a formal context analogous but different to Formal Concept Analysis and Formal Independent Analysis. In this instance, we choose to analyse the information in the incidence table in terms of the partitions induced on the sets of objects and attributes by the same functions of single attributes and objects of the context. These functions constitute a left adjunction between sets of objects and attributes and we later lift this left adjunction to partitions of the objects and attributes. Therefore we refer to this new view onto the formal context as Formal Equivalence Analysis. Rather than looking on the effect of these partitions on set representation, as in Rough Sets, we try to make explicit the information in the context.
\end{abstract}

Keywords: Formal Concept Analysis. Partition lattices. Neighbourhood lattices.

\section{Introduction and Motivation}

The original intent of Wille on creating Formal Concept Analysis (FCA) was, in his own words, an "attempt to unfold lattice-theoretical concepts, results, and methods in a continuous relationship with their surrounding" [15, §1]. In an interesting Final remarks of that seminal work Wille renounces any attempt at exhaustiveness of the lattice restructuring program and recommends: "Besides the interpretation by hierarchies of concepts, other basic interpretations of lattices should be introduced; ..."

Previous work on expanding this program concerned itself with Formal Independence Analysis (FIA), an attempt at seeing the relations of independence between objects, resp. attributes, in a formal context based in their antichains 14. There the analogues of formal concepts are formal tomoi, that capture the absence of partial ordering between objects, resp. attributes, due to their attribute descriptions after the context, resp. object descriptions. A first instance of their usefulness in a particular problem is [13.

The relationship between FCA and Rough Sets is touched upon in many sources. Our perspective on this was radically improved by [5, 6]: some of the traditional closure and interior operators arise from the study of the two adjunctions definable by a binary relation. The main difference between the general field of FCA and Rough Sets is a difference of purpose not of tools.

In this respect Formal Equivalence Analysis (FEA) introduced in this paper-despite dealing with equivalences and partitions, will be mainly concerned with making explicit and organizing the information in a formal context - in the spirit of FCA - and less interested on the constraints imposed by such partitions in approximating sets - as in Rough Sets.

\subsection{The Cognitive Biases of Classification}

Our focus in this paper is to try to model and understand the process of object classification by means of binary attributes.

In a classification system the ontological status of objects and attributes is different with respect to equality:

- If under any criterion (read "attribute") two objects are equivalent, they are considered similar and this is a valuable piece of information. If under all criteria two objects are equivalent, then they are maintained in the same class. Hence we are looking for classes of similar, equivalent objects.

- If two attributes are equal over a subset of entities then they are somehow considered to be correlated 
and this is a valuable piece of information. If under all criteria two attributes are equal then they are redundant and one of them may be ignored. Hence we are looking for classes of redundant attributes.

These biases proceed from the different purposes of classification: objects are considered "given" while attributes are "chosen". In particular, we do not object to attribute clarification or reduction, but we should not try to object-clarify classification contexts unless we want to incur in information loss.

We believe this manner of proceeding is best modelled by a left adjunction but since the emphasis is on equivalence the modelling is better carried out in terms of equivalence relations, or isomorphically, on partitions on sets of objects and attributes.

\subsection{A first approach using FCA}

In this paper we plan to explore the possibility of carrying out the analysis of the information in the context by means of equivalences between objects, dually attributes. Recall that FCA was designed to elicit the hierarchical relations embedded within the formal context by means of the partial order between formal concepts. However, the polars of $\mathrm{FCA}^{1}$ generate quotient sets on the sets of objects and attributes as briefly stated in [7, $\S 1.5]$ : consider the equivalences $\operatorname{ker} \bar{\gamma}$ and $\operatorname{ker} \bar{\mu}$

$$
\begin{aligned}
\left(g_{1}, g_{2}\right) & \in \operatorname{ker} \bar{\gamma} \Longleftrightarrow \bar{\gamma}\left(g_{1}\right)=\bar{\gamma}\left(g_{2}\right) \\
\left(m_{1}, m_{2}\right) & \in \operatorname{ker} \bar{\mu} \Longleftrightarrow \bar{\mu}\left(g_{1}\right)=\bar{\mu}\left(g_{2}\right) .
\end{aligned}
$$

Then the reduced [7] or purified [4] formal context resynthesized by the FCA procedure above is

$$
\mathbb{K}(\mathfrak{B}(G, M, I))=\left(G / \operatorname{ker} \bar{\gamma}, M / \operatorname{ker} \bar{\mu}, I^{\prime}\right)
$$

where $\left([g]_{\operatorname{ker} \bar{\gamma}},[m]_{\operatorname{ker} \bar{\mu}}\right) \in I^{\prime} \Longleftrightarrow g I m$. Note that the simple context construction above for synthesizing $\mathbb{K}$ is important in e.g. categorical accounts of FCA $[3,4]$.

Yet the synthesis capabilities of FCA do not allow to recover the individual elements of such classes: they are simply not present in any lattice $\mathcal{V}$ and can not be "put back" into the context. It is in this sense that we say that FCA has a blind spot for equivalence classes and in general disposes of the information in equivalence relations.

\section{Theory and Methods}

\subsection{The Lattice of Partitions of a Finite Set}

This material stems mostly from [8] and [2, Sections I.8 \& IV.9]. Earlier references are [1, 9. Let $G$ be a set,

\footnotetext{
${ }^{1}$ See Section 2.2 for notational conventions.
}

and call a partition of a set $\pi$ of non-empty ${ }^{2}$, pairwise disjoint subsets of $G$ whose union is $G$. The members of $\pi$ are called the blocks of the partition. A block that is a singleton is called trivial. If $g_{1}$ and $g_{2}$ belong to the same block we write $g_{1} \equiv g_{2}(\pi)$.

Let $\Pi(G)$ denote the set of partitions over $G$. And define the coarsening order:

$$
\pi \leq \sigma \Longleftrightarrow \forall g_{1}, g_{2} \in G, g_{1} \equiv g_{2}(\pi) \Rightarrow g_{1} \equiv g_{2}(\sigma)
$$

that we read as " $\pi$ is less coarse than $\sigma$ ". We have the following result, crucial for our purposes:

Theorem 2.1. [8] Let $G$ be a set. Then $\operatorname{Part}(G)=$ $\langle\Pi(G), \leq\rangle$ is a complete lattice, called the partition lattice (or equivalence lattice of $G$ ) where:

1. The bottom of $\operatorname{Part}(G)$ is $\iota_{G}=\{\{g\} \mid g \in G\}$, the set of trivial blocks.

2. The top of $\operatorname{Part}(G)$ is $\omega_{G}=\{G\}$.

3. The meet of partitions $\left\{\pi_{i} \mid i \in I\right\}$ is defined, for all $g_{1}, g_{2} \in G$ as:

$$
g_{1} \equiv g_{2}\left(\wedge_{i \in I} \pi_{i}\right) \Longleftrightarrow \forall i \in I, g_{1} \equiv g_{2}\left(\pi_{i}\right)
$$

4. The join of partitions $\left\{\pi_{i} \mid i \in I\right\}$ is defined, for all $g_{1}, g_{2} \in G$ as: $g \equiv d\left(\vee_{i \in I} \pi_{i}\right)$ if and only if there is a natural number $n$, a subset $J=\left\{i_{0}, \ldots, i_{n}\right\} \subseteq I$, and $g_{0}, \ldots, g_{n+1} \in G$ such that $g=g_{0}, \ldots, d=g_{n+1}$ and $g_{j}=g_{j+1}\left(\pi_{i_{j}}\right)$, for $0 \leq j \leq n$.

5. The atoms of $\operatorname{Part}(G)$ are the partitions with exactly one non-trivial block and this block has two elements.

6. The co-atoms of $\operatorname{Part}(G)$ are the partitions with exactly two blocks.

7. The covering relation in $\operatorname{Part}(G)$ holds $\pi \prec \sigma$ iff $\sigma$ is the result of replacing two blocks of $\pi$ by their union.

Note that some authors prefer to use the dual order to that defined above, that they call the refinement order.

In the context of FCA, two interesting results due to Birkhoff [2] are:

Proposition 2.2. Let $G$ be a set and consider $\pi \in$ $\Pi(G)$. Then,

1. Its order filter $\uparrow \pi \subseteq \operatorname{Part}(G)$ is isomorphic to the partition lattice of the set $\pi, \uparrow \pi \cong \operatorname{Part}(\pi)$.

2. Its order ideal $\downarrow \pi \subseteq \operatorname{Part}(G)$ is isomorphic to direct product of $\operatorname{Part}(X)$ where $X$ ranges over the non-trivial blocks of $\pi, \downarrow \pi \cong X_{X \in \pi} \operatorname{Part}(X)$.

\footnotetext{
${ }^{2}$ Note that the requisite that $\varnothing$ does not belong to a partition is explicitly requested.
} 
Note that if $X$ is trivial then $\operatorname{Part}(X) \cong \mathbb{1}$ and $\mathbb{1} \times L \cong$ $L \times \mathbb{1} \cong L$.

Example 1. For $G=\{1,2,3,4,5,6\}$ consider partition $\pi_{0}=\{\{1\},\{2,3\},\{4,5,6\}\}$. Its neighbourhood $\uparrow \pi_{0}$ can be seen in Fig. 4 a.

\subsection{The Lattice of Neighbourhood of Objects}

Given a formal context $(G, M, I)$ define the operators:

$$
\begin{aligned}
& (\cdot)_{I}^{\exists}: 2^{G} \rightarrow 2^{M} \\
& X_{I}^{\exists}=\{m \in M \mid(\exists g \in G)[g \in X \wedge g I m]\} \\
& (\cdot)_{I}^{\forall}: 2^{M} \rightarrow 2^{G} \\
& Y_{I}^{\forall}=\{g \in G \mid(\forall m \in M)[g I m \Rightarrow m \in Y)\}
\end{aligned}
$$

These are the span of a set of objects $X_{I}^{\exists}$ as the set of attributes related to some object $g \in X$, and the content of a set of attributes $Y_{I}^{\forall}$ as the set of objects which can be completely described by the attributes in $Y$ [5, 6].

We have the following easy lemma:

Lemma 2.3. Let $\mathbb{K}=(G, M, I)$ be formal context. then:

$$
\begin{aligned}
& X_{I}^{\exists}=\left\{m \in M \mid m^{\downarrow} \cap X \neq \varnothing\right\} \\
& Y_{I}^{\forall}=\left\{g \in G \mid g^{\uparrow} \subseteq Y\right\}
\end{aligned}
$$

and:

$$
X_{I}^{\exists}=\bigcup_{g \in X} g_{I}^{\exists} \quad Y_{I}^{\forall}=\bigcap_{m \in \bar{Y}} \bar{m}_{I}^{\forall}
$$

Proof. The first line and the first half of the second are straightforward. For the last $\overline{Y_{I}^{\forall}}=\left\{g \in G \mid g^{\uparrow} \cap \bar{Y} \neq\right.$ $\varnothing\}=\bigcup_{m \in \bar{Y}}\left\{g \in G \mid g^{\uparrow} \cap m \neq \varnothing\right\}$ therefore

$$
Y_{I}^{\forall}=\bigcap_{m \in \bar{Y}}\left\{g \in G \mid g^{\uparrow} \subseteq \bar{m}\right\}=\bigcap_{m \in \bar{Y}} \bar{m}_{I}^{\forall}
$$

It is also easy to see that $\left(\cdot \frac{\exists}{I},{ }_{I}^{\forall}\right):\left(2^{G}, \subseteq\right) \leftrightharpoons\left(2^{M}, \subseteq\right)$ is a left Galois adjunction. We consider the lattice of its fixed points $\underline{\mathfrak{N}}(G, M, I)$ whose carrier set of neighbour pairs,

$$
\mathfrak{N}(\mathbb{K})=\left\{\langle X, Y\rangle \subseteq \mid X_{I}^{\exists}=Y, Y_{I}^{\forall}=X\right\}
$$

is ordered by $\left(X_{1}, Y_{1}\right) \leq\left(X_{2}, Y_{2}\right) \Longleftrightarrow X_{1} \leq X_{2} \Longleftrightarrow$ $Y_{1} \leq Y_{2}$. Define the mappings

$$
\begin{aligned}
\bar{\gamma}_{I}(\cdot): G \rightarrow \mathfrak{N}(\mathbb{K}) & \bar{\gamma}_{I}(g)=\left\langle\left[g_{I}^{\exists}\right]_{I}^{\forall}, g_{I}^{\exists}\right\rangle \\
\bar{\mu}_{I}(\cdot): M \rightarrow \mathfrak{N}(\mathbb{K}) & \bar{\mu}_{I}(m)=\left\langle\bar{m}_{I}^{\exists},\left[\bar{m}_{I}^{\forall}\right]_{I}^{\exists}\right\rangle
\end{aligned}
$$

Then we can state the:
Theorem 2.4. (Basic theorem of Neighbourhood Lattices, [5]) Let $\mathbb{K}=(G, M, I)$ be a formal context. Then

1. Analysis Part. $\underline{\mathfrak{N}}(\mathbb{K})$ carries a complete lattice structure $\mathfrak{N}(\mathbb{K})=\langle\underline{\mathfrak{N}}(\mathbb{K}), \leq\rangle$ in which infimum and supremum are given by

$$
\begin{aligned}
& \bigwedge_{t \in T}\left(A_{t}, B_{t}\right)=\left(\bigcap_{t \in T} A_{t},\left[\left[\bigcap_{t \in T} B_{t}\right]_{I}^{\forall}\right]_{I}^{\exists}\right) \\
& \bigvee_{t \in T}\left(A_{t}, B_{t}\right)=\left(\left[\left[\bigcup_{t \in T} A_{t}\right]_{I}^{\exists}\right]_{I}^{\forall}, \bigcup_{t \in T} B_{t}\right) .
\end{aligned}
$$

The set $\bar{\gamma}_{I}(G)$ is $\vee$-dense and $\bar{\mu}_{I}(M)$ is $\wedge$-dense.

2. Synthesis part. Conversely, a complete lattice $V$ is isomorphic to $\underline{\mathfrak{N}}(\mathbb{K})$ if and only if there are mappings

$$
\bar{\gamma}: G \rightarrow V \quad \bar{\mu}: M \rightarrow V
$$

such that $\bar{\gamma}(G)$ is join-dense in $V$ and $\bar{\mu}(M)$ is meet-dense in $V$ and $\mathrm{gIm}$ is equivalent to $\bar{\gamma}(g) \not \leq$ $\bar{\mu}(m)$ for all $g \in G$ and all $m \in M$.

This result shows that $\mathfrak{N}(G, M, I)$ is isomorphic to $\underline{\mathfrak{B}}(G, M, \mp)[6]$ - where $\Psi$ is the complement of $I$-but the drawing and interpretation in terms of neighbourhood pairs must differ.

It is well known that the Neighbourhood of objects $\mathfrak{N}(G, M, I)$ may be synthesized as the concept lattice of a context appropriately related to $(G, M, I)[12$. The conceptual synthesis proceeds as follows:

1. Conceptually, start from the complementary context $(G, M, \mp)$.

2. Then as per the recipe, complement the relation and transform $M$ into the set of attribute complements $\bar{M}=\{M \backslash\{m\} \mid m \in M\}$ to obtain derived context $(G, \bar{M}, I)$.

3. Obtain the concept lattice $(G, \bar{M}, I)$ which is isomorphic to $\mathfrak{B}(G, M, I)$ but has complements to attributes instead of attributes.

4. Complement the system of attributes, e.g. $\{\bar{a}, \bar{b}\}=(G \backslash\{a\}) \cap(G \backslash\{b\})$ to obtain a system of open sets.

This is further made clean in the following example.

ExAmple 2. Consider the lattice $\mathcal{L}$ in Fig. 11a that we want to synthesise as the partition lattice of some context. We name the join- and meet-irreducibles as

$$
\mathcal{J}(\mathcal{L})=\{1,6,4,2\} \quad \mathcal{M}(\mathcal{L})=\{a, b, c\}
$$


-notice that the names are unimportant as long as they describe objects and attributes - and then we write the short context $\mathbb{S}(\mathcal{L})=(\mathcal{J}(\mathcal{L}), \mathcal{M}(\mathcal{L}), \leq)$ as shown in Fig. 1 $1 \mathrm{~b}$. It is clear that the concept lattice of this concept is isomorphic to the original one, as seen in Fig. $1 \mathrm{c}$

We then obtain a neighbourhood lattice isomorphic to $\mathcal{L}$ by applying the span and content operators on the complemented context $\mathbb{S}(\mathcal{L})^{-1}$. In this way, a join-dense set of object-spans and a meet dense set of attribute-contents is obtained, as shown in the second columns of Tables $1 \mathrm{a}$ and $1 \mathrm{~b}$, respectively. Note that the meet irreducibles are actually obtained from the negated attribute-concepts as described in the synthesis part of the theorem. Then the neighbourhood lattice $\underline{\mathfrak{N}}(\mathbb{S}(\mathcal{L}))$ of context $\mathbb{S}(\mathcal{L})$ can be reconstructed from the object- and attribute-neighbourhood concepts shown in Table 1 (left columns). Notice that $\underline{\mathfrak{N}}(\mathbb{K})$ has as a bottom $\langle\varnothing, \varnothing\rangle$.

\section{The Mathematical Basis for Formal Equivalence Analysis}

\subsection{Local coarsening and refinement of partitions.}

The following extra operations on lattices will be used in the following.

Definition 3.1. Let $G$ and $G^{\prime}$ be two different sets. Call $A \subseteq G$ saturated with respect to $\pi \in \Pi(G)$ if it is a union of blocks of $\pi$ and define:

\section{The concatenation of two partitions on dif-} ferent sets $G$ and $G^{\prime}$ is an external operation that obtains a partition in the union $G \cup G^{\prime}$. If $\pi=\left\{A_{1}, \ldots, A_{n}\right\}$ and $\pi^{\prime}=\left\{A_{1}^{\prime}, \ldots, A_{n}^{\prime}\right\}$ then $\pi+\pi^{\prime}=\left\{A_{1}, \ldots, A_{n}, A_{1}^{\prime}, \ldots, A_{n}^{\prime}\right\}$.

2. The trace of $\pi$ by $\boldsymbol{a}$ set $A$ as the operation:

$$
(\pi, A) \mapsto \pi_{A}=\left\{A_{1} \cap A, \ldots, A_{n} \cap A\right\}
$$

In the previous definitions, we have the following remarks:

1. Concatenation is associative, and it can always be expressed in terms of joins or meets, e.g. $\pi+\pi^{\prime}=$ $\left(\pi \cup\left\{G^{\prime}\right\}\right) \wedge\left(\pi^{\prime} \cup\{G\}\right)$ in which case it is clearly a commutative, internal operation to $\Pi\left(G \cup G^{\prime}\right)$. We will use it only in this latter meaning. In that case we suppose that $\omega_{\varnothing}+\pi=\iota_{\varnothing}+\pi=\pi$.

2. Concatenation and trace may be made to work as inverses since if $\pi^{\prime \prime}=\pi+\pi^{\prime}$ with $\pi \in \Pi(G), \pi^{\prime} \in$ $\Pi\left(G^{\prime}\right)$ we have that $\left(\pi^{\prime \prime}\right)_{G}=\pi$ and $\left(\pi^{\prime \prime}\right)_{G^{\prime}}=\pi^{\prime}$. In this case, the trace acts as a projection.
With respect to a partition $\pi \in \Pi(G)$ we can locally introduce or dispose of saturated blocks:

Definition 3.2. Let $G$ be a finite set. For a reference $\pi \in \Pi(G), A \subseteq G$ define the partitions:

$$
\text { 1. conflate } A \text { (in } \pi) \text { as } \theta(A)=\pi \vee\left(\omega_{A}+\iota_{\bar{A}}\right)
$$$$
\text { 2. respect } A \text { (in } \pi) \text { as } \kappa(A)=\pi \wedge\left(\omega_{A}+\iota_{\bar{A}}\right)
$$

The following immediate lemma describes in which sense conflate and respect are somehow complementary operations.

Lemma 3.3. Let $G$ be a finite set. For a reference $\pi \in \Pi(G), A \subseteq G$ we have:

$$
\begin{array}{ll}
\theta(G)=\omega_{G} & \kappa(G)=\pi \\
\theta(\varnothing)=\pi & \kappa(\varnothing)=\iota_{G}
\end{array}
$$

\subsection{Finding base partitions for objects and attributes.}

Given a context $(G, M, I)$, define two partitions in the sets of objects and attributes in reference to the left adjunction $\left(\cdot{ }_{I}^{\exists},{ }_{I}^{\forall}\right): 2^{G} \leftrightharpoons 2^{M}$, respectively by:

$$
\begin{aligned}
g_{1}=g_{2}\left(\pi_{0}\right) & \Longleftrightarrow\left(g_{1}\right)_{I}^{\exists}=\left(g_{2}\right)_{I}^{\exists} \\
m_{1}=m_{2}\left(\sigma_{0}\right) & \Longleftrightarrow\left(\bar{m}_{1}\right)_{I}^{\forall}=\left(\bar{m}_{2}\right)_{I}^{\forall}
\end{aligned}
$$

We will refer to the blocks of these partitions in a standard way as

$$
\pi_{0}=\left\{A_{g}\right\}_{g \in G} \quad \sigma_{0}=\left\{B_{m}\right\}_{m \in M}
$$

Note that $\pi_{0} \equiv \operatorname{ker} \bar{\gamma}$ in FCA while the obvious analogue is not necessarily the case for $\pi_{0}$ :

EXAMPLE 2.[Continued] In a reduced context such as $\mathbb{S}(\mathcal{L})$ it is not possible to synthesise the partition lattice we have introduced, since the partitions induced by spans and contents are the finest: $\iota_{\mathcal{J}(\mathcal{L})}$ and $\iota_{\mathcal{M}(\mathcal{L})}$. To describe a lattice of partitions isomorphic to $\mathcal{L}$ we have to introduce partitions whose blocks are nonunitary. For that purpose we create the quadruple context $\mathbb{K}_{2}=(\mathbb{S}(\mathcal{L}) \| \mathbb{S}(\mathcal{L})) /(\mathbb{S}(\mathcal{L}) \| \mathbb{S}(\mathcal{L}))$. Therefore for $G=\mathcal{J}(\mathcal{L}) \cup \mathcal{J}(\mathcal{L})$ and $M=\mathcal{M}(\mathcal{L}) \cup \mathcal{M}(\mathcal{L})^{\prime}$ we have $\mathbb{K}_{2}=(G, M, I)$ for a certain relation $I$ that is built by apposition and subposition of the complementary of the original. In this new context, for $g \in \mathcal{J}(\mathcal{L})$ using the definition of the base partition $\pi_{0}$ of $(9)$ we have $A_{g}=\left\{g, g^{\prime}\right\}$ where $g^{\prime} \in \mathcal{M}(\mathcal{L})^{\prime}$, and analogously for the block $B_{m}$ defined by an $m \in \mathcal{M}(\mathcal{L})$, $B_{m}=\left\{m, m^{\prime}\right\}$.

This does not complicate the representation at all due to the fact that the trace may recover the original partition $\left(\pi_{0}^{\prime \prime}\right)_{G}=\pi_{0}$, and so we may index the partition

\footnotetext{
${ }^{3}$ Naming blocks twice - e.g. for $m^{\prime}=m\left(\sigma_{0}\right)$-is simply absorbed in the set notation for partitions.
} 


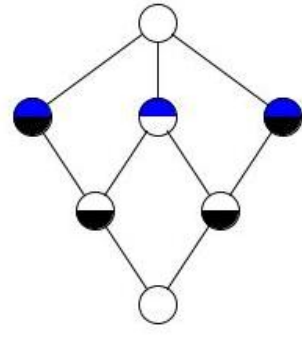

(a) Lattice $\mathcal{L}$

\begin{tabular}{|l||c|c|c|}
\hline $\mathbb{S}(\mathcal{L})^{-1}$ & $\mathrm{a}$ & $\mathrm{b}$ & $\mathrm{c}$ \\
\hline \hline 1 & & $\times$ & $\times$ \\
\hline 2 & $\times$ & & \\
\hline 4 & $\times$ & $\times$ & \\
\hline 6 & & & $\times$ \\
\hline
\end{tabular}

(d) Context $\mathbb{S}(\mathcal{L})^{-1}$

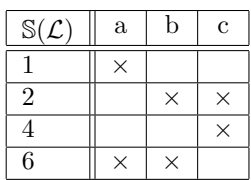

(b) Context $\mathbb{S}(\mathcal{L})$

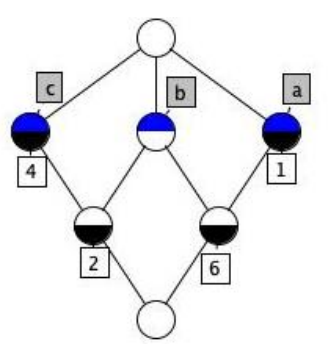

(c) Concept lattice $\underline{\mathfrak{B}}(\mathbb{S}(\mathcal{L}))$

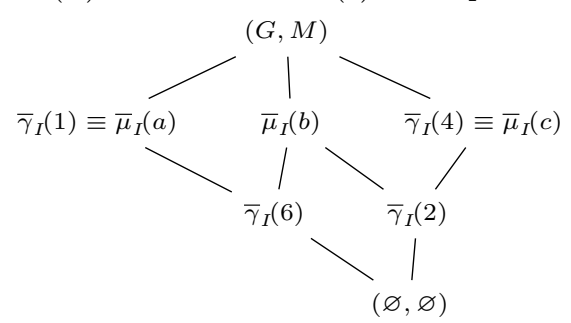

(e) Neighbourhood lattice of $\mathbb{S}(\mathcal{L})$

Figure 1: Lattice $\mathcal{L}$, its short context $\mathbb{S}(\mathcal{L})$, its contrary $\mathbb{S}(\mathcal{L})^{-1}$ and its neighbourhood lattice $\mathfrak{N}(\mathbb{S}(\mathcal{L}))$

\begin{tabular}{c|c|c||c|c}
\hline$g$ & $g_{I}^{\exists}$ (object-spans) & $\left(g_{I}^{\exists}\right)_{I}^{\forall}$ & $\kappa_{G}\left(\left[g_{I}^{\exists}\right]_{I}^{\forall}\right)$ & $\kappa_{M}\left(g_{I}^{\exists}\right)$ \\
\hline 1 & $\{b, c\}$ & $\{1,6\}$ & $\left\{A_{1}, A_{6},\right\}+\iota \overline{A_{1} \cup A_{6}}$ & $\left\{B_{b}, B_{c}\right\}+\iota \overline{B_{b} \cup B_{c}}$ \\
2 & $\{a\}$ & $\{2\}$ & $\left\{A_{2}\right\}+\iota \overline{A_{2}}$ & $\left\{B_{a}\right\}+\iota \overline{B_{a}}$ \\
4 & $\{a, b\}$ & $\{2,4\}$ & $\left\{A_{2}, A_{4}\right\}+\iota \overline{A_{2} \cup A_{4}}$ & $\left\{B_{a}, B_{b}\right\}+\iota \overline{B_{a} \cup B_{b}}$ \\
6 & $\{c\}$ & $\{6\}$ & $\left\{A_{6}\right\}+\iota \overline{A_{6}}$ & $\left\{B_{c}\right\}+\iota \overline{B_{c}}$ \\
\hline
\end{tabular}

(a) Columns 2 and 3: Object concepts of $\underline{\mathfrak{N}}(\mathbb{S}(\mathcal{L}))$

\begin{tabular}{c|c|c|c||c|c}
\hline$m$ & $\bar{m}$ & $\bar{m}_{I}^{\forall}$ (comp. att.-contents) & $\left(\bar{m}_{I}^{\forall}\right)_{I}^{\exists}$ & $\kappa_{G}\left(\bar{m}_{I}^{\forall}\right)$ & $\kappa_{M}\left(\left[\bar{m}_{I}^{\forall}\right]_{I}^{\exists}\right)$ \\
\hline$a$ & $\{b, c\}$ & $\{1,6\}$ & $\{b, c\}$ & $\left\{A_{1}, A_{6}\right\}+\iota \overline{A_{1} \cup A_{6}}$ & $\left\{B_{b}, B_{c}\right\}+\iota \overline{B_{b} \cup B_{c}}$ \\
$b$ & $\{a, c\}$ & $\{2,6\}$ & $\{a, c\}$ & $\left\{A_{2}, A_{6}\right\}+\iota \overline{A_{2} \cup A_{6}}$ & $\left\{B_{a}, B_{c}\right\}+\iota \overline{B_{a} \cup B_{c}}$ \\
$c$ & $\{a, b\}$ & $\{2,4\}$ & $\{a, b\}$ & $\left\{A_{2}, A_{4}\right\}+\iota \overline{A_{2} \cup A_{4}}$ & $\left\{B_{a}, B_{b}\right\}+\iota \overline{B_{a} \cup B_{b}}$ \\
\hline
\end{tabular}

(b) Columns 2, 3 and 4: complemented attribute concepts of $\underline{\mathfrak{N}}(\mathbb{S}(\mathcal{L}))$

Table 1: Object- and attribute-induced neighbourhood concepts from $\mathfrak{N}(\mathbb{S}(\mathcal{L})$ ) and their induced partitions (see $\S 2$

on the originals objects to univocally represent their classess $\pi_{0}^{\prime \prime}=\sum_{g \in G} \omega_{A_{g}}$.

\subsection{A left adjunction on partitions}

To establish an isomorphism with $\mathfrak{R}(G, M, I)$ we use two "respect" operations, one on each set of partitions $\operatorname{Part}(G)$ and $\operatorname{Part}(M)$ where the reference partitions are $\pi_{0}$ and $\sigma_{0}$ for

$$
\kappa_{G}(X)=\pi_{0} \wedge\left(\omega_{X}+\iota_{\bar{X}}\right) \quad \kappa_{M}(Y)=\sigma_{0} \wedge\left(\omega_{Y}+\iota_{\bar{Y}}\right)
$$

Note that for all $X \subseteq G$ we have $\kappa_{G}(X) \leq \pi_{0}$ and analogously in the partition lattice of attributes. In fact we have the lemma:

Lemma 3.4. Let the operators $\kappa_{G}(\cdot)$ and $\kappa_{M}(\cdot)$ as above. Then,

$$
\begin{gathered}
g_{1} \equiv g_{2}\left(\kappa_{G}(X)\right) \text { iff }\left\{\begin{array}{l}
g_{1}=g_{2} \text { or } \\
\left(g_{1}\right)_{I}^{\exists}=\left(g_{2}\right)_{I}^{\exists} \text { and } g_{1} \in X
\end{array}\right. \\
m_{1} \equiv m_{2}\left(\kappa_{M}(Y)\right) \text { iff }\left\{\begin{array}{l}
m_{1}=m_{2} \text { or } \\
\left(\bar{m}_{1}\right)_{I}^{\forall}=\left(\bar{m}_{2}\right)_{I}^{\forall} \text { and } m_{1} \in Y
\end{array}\right.
\end{gathered}
$$

We want to design the construction of a lattice $L \subseteq$ $\Pi(G) \times \Pi(M)$ whose join- and meet-irreducibles are one-to-one with those of $\mathfrak{N}(G, M, I)$. Then we would 
inject basic object and attribute partitions as:

$$
\begin{aligned}
& \bar{\gamma}_{\Pi}(\cdot): G \rightarrow \operatorname{Part}(G) \times \operatorname{Part}(M) \\
& g \mapsto \bar{\gamma}_{\Pi}(g)=\left(\kappa_{G}\left(\left(g_{I}^{\exists}\right)_{I}^{\forall}\right), \kappa_{M}\left(g_{I}^{\exists}\right)\right) \\
& \bar{\mu}_{\Pi}(\cdot): M \rightarrow \operatorname{Part}(G) \times \operatorname{Part}(M) \\
& m \mapsto \bar{\mu}_{\Pi}(m)=\left(\kappa_{G}\left(\bar{m}_{I}^{\forall}\right), \kappa_{M}\left(\left(\bar{m}_{I}^{\forall}\right)_{I}^{\exists}\right)\right)
\end{aligned}
$$

For that purpose consider the following.

Theorem 3.5. The function $\kappa: \mathfrak{N}(\mathbb{K}) \rightarrow \operatorname{Part}(G) \times$ $\operatorname{Part}(M)$ with $\kappa(\langle X, Y\rangle)=\left\langle\kappa_{G}(X), \kappa_{M}(Y)\right\rangle$ is a $\wedge$ homomorphism.

Therefore the following is immediate.

Corollary 3.6. The image $\kappa(\mathfrak{N}(\mathbb{K}))$ is a closure system within the set $\operatorname{Part}(G) \times \operatorname{Part}(M)$, which is the lattice $\mathfrak{P}(\mathbb{K})$.

Note that $\bar{\gamma}_{\Pi}(g)=\kappa\left(\bar{\gamma}_{I}(g)\right)$ and $\bar{\mu}_{\Pi}(m)=\kappa\left(\bar{\mu}_{I}(m)\right)$ where $\bar{\gamma}_{I}(\cdot)$ and $\bar{\mu}_{I}(\cdot)$ come from $(5)$, whence:

Corollary 3.7. The set $\bar{\gamma}_{\Pi}(G)=\kappa\left(\bar{\gamma}_{I}(G)\right)$ is $\vee$-dense in $\mathfrak{P}(\mathbb{K})$ and $\left.\bar{\mu}_{\Pi}(M)=\kappa\left(\bar{\mu}_{I}(M)\right)\right)$ is $\wedge$-dense in $\mathfrak{P}(\mathbb{K})$.

The following clarifies why it is important to have nonunitary blocks in partitions.

Corollary 3.8. If $\mathbb{K}$ has all of its columns and rows duplicated, then $\kappa$ is injective, and therefore, $\mathfrak{N}(\mathbb{K})$ and $\mathfrak{P}(\mathbb{K})$ are isomorphic.

In that case, furthermore, $\mathfrak{P}(\mathbb{K})$ is isomorphic to the usual concept lattice associated to the opposite context $\mathbb{K}_{0}^{-1}=(G, M, I)$.

In particular this is the case if we define a left adjunction between $\Pi(G)$ and $\Pi(M)$. So consider the projection of tuples over pairs of partitions: if $c=(\pi, \sigma) \in$ $\Pi(G) \times \Pi(M)$, then $\sigma_{c}=\sigma$ and $\pi_{c}=\pi$. Now for partitions of objects $G$ and attributes $M$ define the functions $(\cdot)_{\Pi}^{\exists}: \Pi(G) \rightarrow \Pi(M)$ and $(\cdot)_{\Pi}^{\forall}: \Pi(M) \rightarrow \Pi(G)$ :

$$
\begin{aligned}
\pi_{\Pi}^{\exists} & =\bigvee\left\{\sigma_{c} \mid c \in \bar{\mu}_{\Pi}(M), \pi \geq \pi_{c}\right\} \\
\sigma_{\Pi}^{\forall} & =\bigwedge\left\{\pi_{c} \mid c \in \bar{\gamma}_{\Pi}(G), \sigma \leq \sigma_{c}\right\}
\end{aligned}
$$

Lemma 3.9. $\left(\cdot \frac{\exists}{\Pi}, \frac{\forall}{\Pi}\right)$ : $\operatorname{Part}(G) \leftrightharpoons \operatorname{Part}(M)$ is a left adjunction between the lattices of partitions of objects and attributes.

We can finally state the:

Theorem 3.10. (Basic theorem of formal equivalence analysis)

1. Analysis phase. Given a formal context $\mathbb{K}=$ $(G, M, I)$,

(a) The operators form a left adjunction whose formal equivalences or partitions are the pairs $(\pi, \sigma)$ such that $\pi_{\Pi}^{\exists}=\sigma \Longleftrightarrow \sigma_{\Pi}^{\forall}=\pi$ (b) The set of formal partitions $\mathfrak{P}(\mathbb{K})$ with the relation $\left(\pi_{1}, \sigma_{1}\right) \leq\left(\pi_{2}, \sigma_{2}\right)$ iff $\pi_{1} \leq$ $\pi_{2}$ iff $\sigma_{1} \leq \sigma_{2}$ is a complete lattice, which is called the partition lattice of $\mathbb{K}$ and denoted $\underline{\mathfrak{P}}(\mathbb{K})$, with infima and suprema given by:

$$
\begin{aligned}
& \bigwedge_{t \in T}\left(\pi_{t}, \sigma_{t}\right)=\left(\bigwedge_{t \in T} \pi_{t},\left[\left(\bigwedge_{t \in T} \sigma_{t}\right)_{\Pi}^{\forall}\right]_{\Pi}^{\exists}\right) \\
& \left.\bigvee_{t \in T}\left(\pi_{t}, \sigma_{t}\right)=\left(\left[\bigvee_{t \in T} \pi_{t}\right)_{\Pi}^{\exists}\right]_{\Pi}^{\forall}, \bigvee_{t \in T} \sigma_{t}\right)
\end{aligned}
$$

(c) The mappings in 13$) \bar{\gamma}_{\Pi}(\cdot): G \rightarrow \mathfrak{P}(\mathbb{K})$ and $\bar{\mu}_{\Pi}(\cdot): M \rightarrow \mathfrak{P}(\mathbb{K})$ are such that $\bar{\gamma}_{\Pi}(G)$ is $\vee$ dense in $\mathfrak{P}(\mathbb{K}), \bar{\mu}_{\Pi}(M)$ is $\wedge$-dense in $\mathfrak{P}(\mathbb{K})$.

2. Synthesis phase. Given a complete lattice $\mathbb{L}=$ $\langle L, \leq\rangle$

(a) $\mathbb{L}$ is isomorphic th $\mathfrak{P}(G, M, I)$ if and only if there are mappings $\bar{\gamma}: G \rightarrow L$ and $\bar{\mu}: M \rightarrow$ $L$ such that

- $\bar{\gamma}(G)$ is $\vee$-dense in $\mathbb{L}, \bar{\mu}(M)$ is $\wedge$-dense in $\mathbb{L}$, and

- $g I m$ is equivalent to $\bar{\gamma}(g) \nsupseteq \bar{\mu}(m)$ for all $g \in G$ and all $m \in M$.

(b) In particular, $\mathbb{L} \cong \mathfrak{P}(L, L, \nsupseteq)$ and, if $L$ is finite, $\mathbb{L} \cong \mathfrak{P}(J(\mathbb{L}), M(\mathbb{L}), \nsupseteq)$ where $M(\mathbb{L})$ and $J(\mathbb{L})$ are the sets of meet- and joinirreducibles, respectively, of $\mathbb{L}$.

ExAMPLE 2.[Continued] We will finish this example by synthesising a partition lattice isomorphic to the original one.

To obtain the object and attribute partition-concepts we use the table of object spans and attribute contents to generate partitions systematically related to them, as explained above. In particular for this example, we obtain the object partition concepts $\bar{\gamma}_{\Pi}(g), g \in G$ and the attribute partition concepts $\bar{\mu}_{\Pi}(\bar{m}), m \in M$ seen in columns $4-5$ of Tables $1 \mathrm{a}$, and $1 \mathrm{~b}$, respectively.

We then construct a partition lattice by completing the order between them, which is isomorphic to that of the object neighbourhood lattice. Figure 2 shows this lattice of partitions annotated with partition concepts isomorphic to $\mathcal{L}$.

ExAmple 3.[Context Analysis] In this basic example we start with a context and carry out its formal equivalence analysis until obtaining its partition lattices.

Consider the formal context of Fig. 3 a and the spans of objects and the contents of complemented attributes as in Tables $3 \mathrm{a}$ and $\mathrm{b}$.

\footnotetext{
${ }^{4}$ Read can be built as.
} 


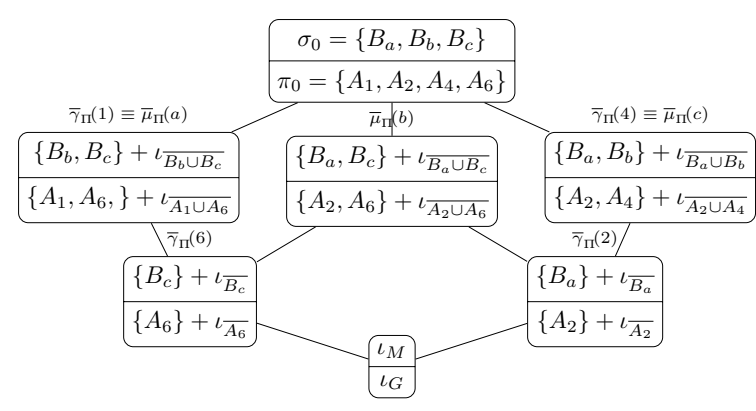

Figure 2: Partition Neighbourhood lattice $\mathfrak{P}\left(\mathbb{K}_{2}\right)$.

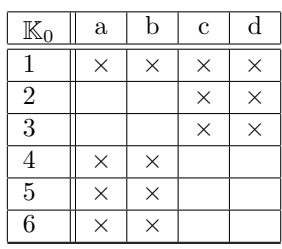

(a) Context $\mathbb{K}_{0}$

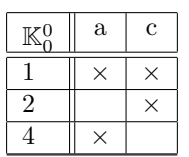

(b) Context $\mathbb{K}_{0}^{0}$
Figure 3: Context $\mathbb{K}_{0}$, its clarified context $\mathbb{K}_{0}^{0}$

Notice that the object and attribute partitions are $\pi_{0}=\{1,23,456\}$ and $\sigma_{0}=\{a b, c d\}$. For convenience while working out the left adjunction concepts we have also placed the clarified context $\mathbb{K}_{0}^{0}=$ $\left(G / \pi_{0}, M / \sigma_{0}, I_{0}\right)$ in Fig. $3 \mathrm{~b}$. This entails that we have named the blocks of the partition as $A_{1}=\{1\}, A_{2}=$ $\{2,3\}, A_{4}=\{4,5,6\}$ and $B_{a}=\{a, b\}, B_{c}=\{c, d\}$ so $\pi_{0}=\left\{A_{1}, A_{2}, A_{4}\right\}$ and $\sigma_{0}=\left\{B_{a}, B_{c}\right\}$.

The neighbourhood $\uparrow \pi_{0}$ is depicted in Fig. 4 a and $\uparrow \sigma_{0}$ in Fig. $4 \mathrm{~b}$. Within the downset lattices $\downarrow \pi_{0}$ and $\downarrow \sigma_{0}$ we may find enough partitions to create the partition lattice of the context. Figure 5 shows the lattice of partitions $\mathfrak{P}\left(\mathbb{K}_{0}\right)$ annotated with partition concepts. For the description of the components of the concepts see Tables $3 \mathrm{a} a$ and $\mathrm{b}$.

\begin{tabular}{c|c|c||c|c}
\hline$g$ & $g_{I}^{\exists}$ & $\left(g_{I}^{\exists}\right)_{I}^{\forall}$ & $\kappa_{G}\left(\left[g_{I}^{\exists}\right]_{I}^{\forall}\right)$ & $\kappa_{M}\left(g_{I}^{\exists}\right)$ \\
\hline 1 & $\{a, b, c, d\}$ & $\{1,2,3,4,5,6\}$ & $\pi_{0}$ & $\sigma_{0}$ \\
2 & $\{c, d\}=B_{c}$ & $\{2,3\}=A_{2}$ & $\left\{A_{2}\right\}+\iota \overline{A_{2}}$ & $\left\{B_{c}\right\}+\iota \overline{B_{c}}$ \\
4 & $\{a, b\}=B_{a}$ & $\{4,5,6\}=A_{4}$ & $\left\{A_{4}\right\}+\iota \overline{A_{4}}$ & $\left\{B_{a}\right\}+\iota \overline{B_{a}}$ \\
\hline
\end{tabular}

(a) Object concepts of $\underline{\mathfrak{R}}\left(\mathbb{K}_{0}\right)$

\begin{tabular}{c|c|c|c||c|c}
\hline$m$ & $\bar{m}$ & $\bar{m}_{I}^{\forall}$ & $\left(\bar{m}_{I}^{\forall}\right)_{I}^{\exists}$ & $\kappa_{G}\left(\bar{m}_{I}^{\forall}\right)$ & $\kappa_{M}\left(\left[\overline{m_{I}}\right]_{I}^{\exists}\right)$ \\
\hline $\mathrm{a}$ & $\{b, c, d\}$ & $\{2,3\}$ & $\{c, d\}$ & $\left\{A_{2}\right\}+\iota \overline{A_{2}}$ & $\left\{B_{c}\right\}+\iota \overline{B_{c}}$ \\
$\mathrm{~b}$ & $\{a, c, d\}$ & $\{2,3\}$ & $\{c, d\}$ & $\left\{A_{2}\right\}+\iota \overline{A_{2}}$ & $\left\{B_{c}\right\}+\iota \overline{B_{c}}$ \\
$\mathrm{c}$ & $\{a, b, d\}$ & $\{4,5,6\}$ & $\{a, b\}$ & $\left\{A_{4}\right\}+\iota \overline{A_{4}}$ & $\left\{B_{a}\right\}+\iota \overline{B_{a}}$ \\
$\mathrm{~d}$ & $\{a, b, c\}$ & $\{4,5,6\}$ & $\{a, b\}$ & $\left\{A_{4}\right\}+\iota \overline{A_{4}}$ & $\left\{B_{a}\right\}+\iota \overline{B_{a}}$ \\
\hline
\end{tabular}

(b) Attribute concepts of $\underline{\mathfrak{N}}\left(\mathbb{K}_{0}\right)$

Table 2: Object- and attribute-concepts of the neighbourhood of objects lattice of $\mathbb{K}_{0}$.

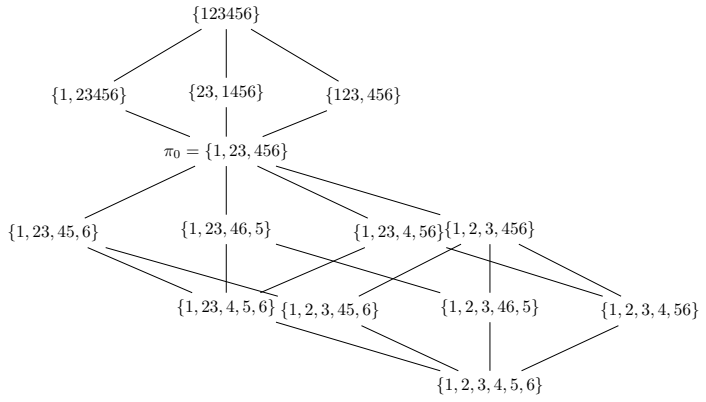

(a) Neighbourhood of $\pi_{0}=\{1,23,456\}$.

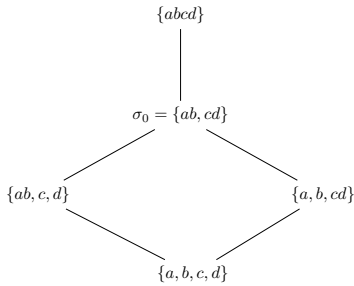

(b) Neighbourhood of $\sigma_{0}=\{a b, c d\}$.

Figure 4: Neighbourhoods of $\pi_{0}$ and $\sigma_{0}$ on $\mathbb{K}_{0}$.

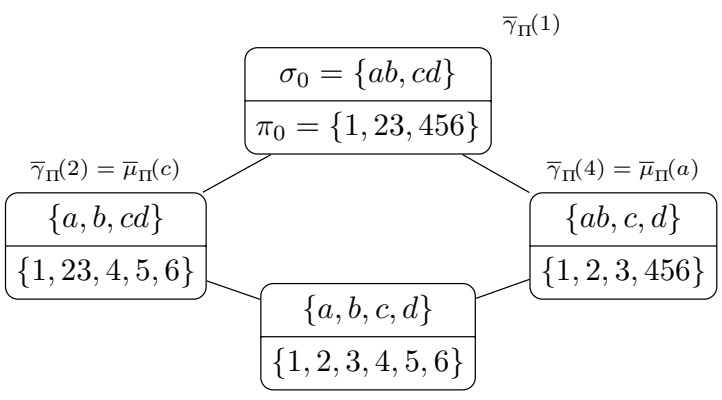

Figure 5: Partition lattice $\mathfrak{P}\left(\mathbb{K}_{0}\right)$.

\subsection{Discussion}

Regarding possible applications of Formal Analysis (FCA, FIA and FEA) to classification:

- An initial FEA would reduce the size complexity of the classification system by aggregating those objects and attributes that have similar descriptions in terms of each mode. The measure e.g. cardinalities - of the classes would provide a basis for providing probability measures for labels taken as random variables. Later in the analysis, partitions in an order relationship would describe coarsenings or refinements of the labels that changed their probabilities.

- A following FIA would allow us to partition the initial problem into several subproblems ameliorating later issues like inference, estimation, etc.

- A final FCA would provide an analysis for dependencies between objects or attributes in terms of associations, rules, etc. 
Of course, this manner of proceeding belongs in Exploratory Data Analysis (EDA) [10] which propounds cycles of exploration and inferencing, hence these phases of analysis could be iterated, mingled or carried in parallel. We have already discussed at length the "affordances" of FCA for EDA [11. In this light, the presence of FIA and FEA are two more tools at the disposal of practitioners, providing the systematic approach that FCA pioneered. On this light, we might wonder whether there are any more Formal XXX Analyses waiting to be modelled.

\section{Conclusions}

We have presented an alternate way to look at the information contained in a formal context than that provided by FCA [7] and FIA [14. In the present instance we look at the equivalences between objects and attributes in terms of the partitions induced by the incidence relation, an endeavour that we have named Formal Equivalence Analysis.

Impelled by the dissimilar role of objects and attributes in cognition, we re-visited the notion of left adjunction arising from binary relation and were able to define an operator that encodes the distinctions among objects and attributes encoded in the concepts in a neigbourhood of objects lattice. With these operators we have been able to obtain an analogue of the Fundamental Theorem for FEA.

It is our belief that the approach provided by FCA, FIA and FEA goes a long way towards fulfilling Wille's program cited in the introduction and we intend to explore it further in future works.

\section{Acknowledgement}

The researchers were partially supported for this work by the Spanish Government-AEI projects TEC2017-84395-P (CPM, FVA), TIN2017-89023-P (PC), TIN2015- 70266-C2-P-1 and PGC2018-095869B-I00 (MOA).

\section{References}

[1] G. Birkhoff, On the Structure of Abstract Algebras, Mathematical Proceedings of the Cambridge Philosophical Society 31 (4) (1935) 433454 .

[2] G. Birkhoff, Lattice theory, American Mathematical Society, 1967.

[3] K. Deiters, M. Erné, Negations and contrapositions of complete lattices, Discrete Mathematics 181 (1-3) (1998) 91-111.

[4] K. Deiters, M. Erné, Sums, products and nega- tions of contexts and complete lattices, Algebra Universalis 60 (4) (2009) 469-496.

[5] I. Düntsch, G. Gediga, Modal-style operators in qualitative data analysis, Tech. Rep. CS-02-15, Department of Computer Science, Brock University, St. Catharines, Ontario, Canada (May 2002).

[6] I. Düntsch, G. Gediga, Approximation operators in qualitative data analysis, in: Theory and Applications of Relational Structures as Knowledge Instruments, Vol. 2929 of Lecture Notes in Computer Science, Springer, 2003, pp. 214-230.

[7] B. Ganter, R. Wille, Formal Concept Analysis: Mathematical Foundations, Springer, Berlin, Heidelberg, 1999.

[8] G. Gratzer, General Lattice Theory, Birkhauser, 2003.

[9] O. Ore, Theory of equivalence relations, Duke Mathematical Journal 9 (3) (1942) 573-627.

[10] J. W. Tukey, Exploratory data analysis, AddisonWesley, 1977.

[11] F. J. Valverde-Albacete, J. M. GonzálezCalabozo, A. Peñas, C. Peláez-Moreno, Supporting scientific knowledge discovery with extended, generalized formal concept analysis, Expert Systems with Applications 44 (2016) 198 - 216.

[12] F. J. Valverde-Albacete, C. Peláez-Moreno, Extending conceptualisation modes for generalised Formal Concept Analysis, Information Sciences 181 (2011) 1888-1909.

[13] F. J. Valverde-Albacete, C. Peláez-Moreno, I. P. Cabrera, P. Cordero, M. Ojeda-Aciego, A Data Analysis Application of Formal Independence Analysis, in: Concept Lattices and their Applications (CLA 2018), 2018, pp. 1-12.

[14] F. J. Valverde-Albacete, C. Peláez-Moreno, I. P. Cabrera, P. Cordero, M. Ojeda-Aciego, Formal Independence Analysis, in: Information Processing and Management of Uncertainty in Knowledge-Based Systems: Applications (IPMU 2018), no. 853 in CCIS, Springer International Publishing, 2018, pp. 596-608.

[15] R. Wille, Restructuring lattice theory: an approach based on hierarchies of concepts, in: Ordered sets (Banff, Alta., 1981), Reidel, DordrechtBoston, Mass., 1982, pp. 445-470. 